\title{
Dermoscopic Features of Hyperpigmented Dots in Crista Cutis in Two Siblings in a Japanese Family with Inherited Acanthosis Nigricans
}

\author{
Shusuke Uchida ${ }^{1}$, Naoki Oiso ${ }^{1 *}$, Tamio Suzuki ${ }^{2}$, Akira Kawada ${ }^{1}$ \\ ${ }^{1}$ Department of Dermatology, Faculty of Medicine, Kinki University, Osaka, Japan; ${ }^{2}$ Department of Dermatology, School of Medi- \\ cine, Yamagata University, Yamagata, Japan. \\ Email: "naoiso@med.kindai.ac.jp
}

Received September $27^{\text {th }}, 2012$; revised October $26^{\text {th }}, 2012$; accepted November $7^{\text {th }}, 2012$

\begin{abstract}
Acanthosis nigricans is characterized by papillomatous brownish lesions mainly in the intertriginous areas. We used dermoscopy to examine such lesions in a family with acanthosis nigricans. The dermoscopic images showed an aberrant skin structure of linear crista cutis and sulcus cutis, and hyperpigmented dots in crista cutis. The hyperpigmented dots, which could not be seen with the naked eyes, may contribute to the color of the pigmented skin. Dermoscopy can be useful for evaluating disorders involving the structure of area cutanea and a change in skin color.
\end{abstract}

Keywords: Dermoscopy; Acanthosis Nigricans; Hyperpigmented Dots; Crista Cutis; Sulcus Cutis

\section{Introduction}

Acanthosis nigricans (AN) can be present in the following forms: inherited, obesity-associated, autoimmune-related, drug-induced, and malignancy-associated. Inherited $\mathrm{AN}$ is a heterogeneous disorder, sub-classified into non-syndromic and syndromic forms [1]. AN is characterized by papillomatous brownish lesions mainly in the intertriginous areas, such as the axillae, neck, genital and submammary regions. The non-syndromic form is present at birth or develops during childhood. Wider regions, including almost the entire skin, may be affected in some hereditary cases.

\section{Case Report}

A 12-year-old Japanese woman visited us with asymptomatic aberrant skin color and structure. The abnormalities had been noticed during infancy. She had no other congenital malformations or delays in mental and physiccal development. The proband's father and her 11-yearold brother had similar abnormal skin color and structure. A physical examination of the proband revealed asymptomatic and hyperpigmented papillomatous lesions over almost the entire body, especially in the intertriginous areas, such as the axillae and the neck (Figure 1(a)). The younger brother showed a similar clinical phenotype (Figure 1(b)). Dermoscopy of the proband's neck show-

${ }^{*}$ Corresponding author. ed linear crista cutis and sulcus cutis (Figure 1(c)). Focal hyperpigmented dots were present in the crista cutis. Dermoscopy of the brother's neck revealed a similar aberrant skin structure with focal pigmented dots (Figure 1(d)). Unfortunately, we could not verify the origin of focal pigmented dots and precise structure of papillomatous projections with histopathological specimens, as the parents did not agree with biopsies.

\section{Discussion}

Dermoscopic images in two siblings with non-syndromic hereditary AN identified hyperpigmented dots that could not be seen with the naked eyes. The dermoscopic features of linear crista cutis would reflect the papillomatous projections of the dermis. We could not take biopsy specimens, even though histopathologic specimens are essential to confirm the origin of the hyperpigmented dots, i.e. increased melanin granules per melanocytes, an increased number of melanin contents-containing keratinocytes, or some other non-melanin-associated pigmentation.

Syndromic acanthosis nigricans is caused by specific activating mutations in FGFR2 and FGFR3 [1]. FGFR2b, the form expressed in the epidermis, mediates FGF signals to RAS-RAF-MAPK and phosphoinositide-3 kinase signaling cascades. Epidermal nevi represent mosaic forms of syndromic AN (FGFR3) and of neuro-cardio-facial-cutaneous syndromes (HRAS, NRAS, and KRAS), a 


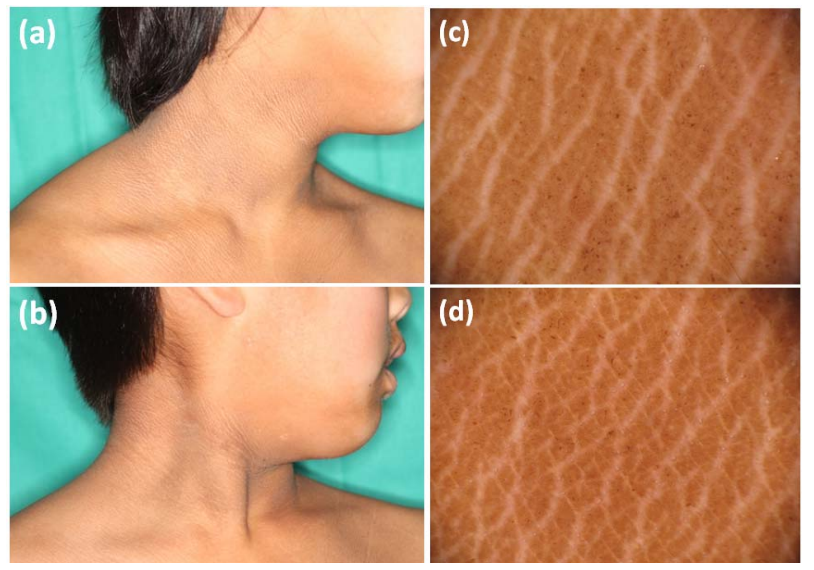

Figure 1. (a) A clinical picture of a 12-year-old Japanese woman; (b) A clinical figure of her 11-year-old Japanese brother; (c) A dermoscopic image of the neck of the 12year-old Japanese woman. It is characterized by miniature hyperpigmented dots and aberrant crista cutis and sulcus cutis. The dermoscopic image of the proband is more highly magnified; (d) A dermoscopic illustration of her 11-year-old Japanese brother. It showed similar hyperpigmented dots and aberrant crista cutis and sulcus cutis.

group of heterogeneous neurodevelopmental disorders including LEOPARD syndrome and neurofibromatosis type $1[2,3]$. The dots in dermoscopic images are darker brown, suggesting that it might correspond to superficial pigmentation. We speculated that the hyperpigmented spots could develop along similar pathways to lentiginosis in LEOPARD syndrome or café-au-lait spots in neurofibromatosis type 1 . The hyperpigmented dots, which could not be seen with the naked eyes, may contribute to the color of the pigmented skin.

With dermoscopy in two siblings, we confirmed hyperpigmented dots and aberrant structure of crista cutis and sulcus cutis. Dermoscopy is useful for evaluating the disorders involving structure of crista cutis and sulcus cutis with a change of color as shown in a case of generalized Dowling-Degos disease [4] or in a variant of linear atrophoderma of Moulin [5].

We reported dermoscopic features in two siblings in a family with inherited non-syndromic AN. Further study with histopathologic correlations of dermoscopic findings in AN should be addressed to recognize more precise pathogenesis.

\section{REFERENCES}

[1] D. R. Berk, E. B. Spector and S. J. Baylies, "Familial Acanthosis Nigricans Due to K650T FGFR3 Mutation," Archive of Dermatology, Vol. 143, No. 9, 2007, pp. 11531156. doi:10.1001/archderm.143.9.1153

[2] C. Hafner, A. Toll, S. Gantner, A. Mauerer, I. Lurkin, F. Acquadro, A. Fernández-Casado, E. C. Zwarthoff, W. Dietmaier, E. Baselga, E. Parera, A. Vicente, A. Casanova, J. Cigudosa, T. Mentzel, R. M. Pujol, M. Landthaler and F. X. Real, "Keratinocytic Epidermal Nevi Are Associated with Mosaic RAS Mutations," Journal of Medical Genetics, Vol. 49, No. 4, 2012, pp. 249-253. doi:10.1136/jmedgenet-2011-100637

[3] S. Yu and W. D. Graf, "BRAF Gene Deletion Broadens the Clinical Spectrum Neuro-Cardio-Facial-Cutaneous Syndromes," Journal of Child Neurology, Vol. 26, No. 12, 2011, pp. 1593-1596. doi:10.1177/0883073811413830

[4] S. Geissler, D. Dyall-Smith, B. Coras, S. Guther, B. Peters and W. Stolz, "Unique Brown Star Shape on Dermatoscopy of Generalized Dowling-Degos Disease," Australasian Journal of Dermatology, Vol. 52, No. 2, 2011, pp. 151-153. doi:10.1111/j.1440-0960.2011.00736.x

[5] N. Oiso, M. Kimura, T. Itoh and A. Kawada, "Variant of Linear Atrophoderma of Moulin: Hyper- and Hypopigmented Linear Atrophoderma with Aberrant Area Cutanea and Lentiginosis Following the Lines of Blaschko," The Journal of Dermatology, Vol. 39, No. 12, 2012, pp. 1097-1099. doi:10.1111/j.1346-8138.2012.01627.x 